April 1994

PUPT-1462

hep-th/9405077

\title{
Probability Density, Diagrammatic Technique, and Epsilon Expansion in the Theory of Wave Turbulence
}

\author{
V. Gurarie G $^{\dagger}$ \\ Department of Physics \\ Princeton University \\ Princeton, NJ 08544
}

\begin{abstract}
We apply the methods of Field Theory to study the turbulent regimes of statistical systems. First we show how one can find their probability densities. For the case of the theory of wave turbulence with four-wave interaction we calculate them explicitly and study their properties. Using those densities we show how one can in principle calculate any correlation function in this theory by means of direct perturbative expansion in powers of the interaction. Then we give the general form of the corrections to the kinetic equation and develop an appropriate diagrammatic technique. This technique, while resembling that of $\varphi^{4}$ theory, has many new distinctive features. The role of the $\epsilon=d-4$ parameter of $\varphi^{4}$ theory is played here by the parameter $\kappa=\beta+d-\alpha-\gamma$ where $\beta$ is the dimension of the interaction, $d$ is the space dimension, $\alpha$ is the dimension of the energy spectrum and $\gamma$ is the "classical" wave density dimension. If $\kappa>0$ then the Kolmogorov index is exact, and if $\kappa<0$ then we expect it to be modified by the interaction. For $\kappa$ a small negative number, $\alpha<1$ and a special form of the interaction we compute this modification explicitly with the additional assumption of the irrelevance of the IR divergencies which still needs to be verified.
\end{abstract}

\footnotetext{
† gurarie@puhep1.princeton.edu
} 


\section{Introduction}

The theory of wave turbulence studies the stationary states of the statistical classical (not quantum) system consisting of waves with a small interaction. (on the theory of wave turbulence see ref. [1] and references therein). Its Hamiltonian can be written down in the following form

$$
H=\sum_{p} \omega_{p} a_{p}^{\dagger} a_{p}+\sum_{p_{1} p_{2} p_{3} p_{4}} \lambda_{p_{1} p_{2} p_{3} p_{4}} a_{p_{1}}^{\dagger} a_{p_{2}}^{\dagger} a_{p_{3}} a_{p_{4}}
$$

It is just a collection of waves with the energy spectrum $\omega_{p}$ and the four wave inter-

action $\lambda_{p_{1} p_{2} p_{3} p_{4}}$ with the evident properties $\lambda_{p_{1} p_{2} p_{3} p_{4}}=\lambda_{p_{2} p_{1} p_{3} p_{4}}=\lambda_{p_{3} p_{4} p_{1} p_{2}}$. Let us note that this Hamiltonian conserves the total wave number

$$
N=\sum_{p} a_{p}^{\dagger} a_{p}
$$

The simplest stationary state of this system is a thermodynamic equilibrium and its probability density is given by the well-known Gibbs distribution $\exp \left(-\frac{H+\mu N}{T}\right)$. The basic property of thermodynamic equilibrium is that the detailed balance principle is satisfied. There are as many waves going from the wave-number $p_{1}$ to $p_{2}$ as there are ones going back. However there are other stationary states where that principle is not satisfied, or in other words, while the total number of waves coming to the given wave number $p$ is zero, there is a flux of waves through the system. Which state will be chosen by the system depends on the external conditions. If it interacts with a heat bath satisfying the detailed balance principle, it will soon settle into the thermodynamic equilibrium. If, on the other hand, the heat bath is so special that it injects waves to the system at one wave number and removes them at a different one, then the system will necessarily choose one of those extra states. A simple example of the latter case is the waves on the surface of water which are injected by, for example, the ship, at wave lengths of the order of ship length, and are dissipated at much smaller lengths by viscosity.

The theory of turbulence concerns itself with studying the "flux" states as the thermodynamic equilibrium has already been studied in great details by Statistical Physics. The standard hydrodynamics turbulence is also the example of the flux states since here we have a very complicated motion of liquid with a stationary probability distribution which is characterized by the flux of energy or other conserved quantities through various scales of vertex motion. 
While the existence of those extra states was known for a long time and they were analyzed with the aid of kinetic equations (ref. [1]), the explicit form of the probability distribution was never found. But let us remember that the Liouville theorem of the statistical mechanics tells us that the probability distribution of any system should be an additive integral of motion. The next step is usually to assume the system doesn't have any additive integrals of motion different from the energy and the particle (or wave) number. That's how Gibbs distribution is obtained. But in fact any dynamical system has as many integrals of motion as the number of its degrees of freedom. Really, any initial conditions expressed in terms of the changing variables give us a formal integral of motion. The important role such integrals play in the description of the flux-states of the statistical systems was first noticed by A. Polyakov (ref. [2]). Essentially such probability distributions mean that we prepared first a statistical ensemble with the probability $\rho(q, p)$ where $q$ and $p$ are the initial coordinates and momenta respectively. Then we follow its time development. A correlation function of some quantity $X(q, p)$ at the moment $t=0$ is given by

$$
\int d q d p X(q, p) \rho(q, p)
$$

To find this function at a later time $t$ we need to use the solutions of the equations of motion, $p(t)$ and $q(t)$, to express the initial conditions in terms of the values of the coordinates and the momenta at a later time $t, q(q(t), p(t), t)$ and $p(q(t), p(t), t)$. The correlation function will then be given by

$$
\int d q d p X(q(t), p(t)) \rho(q, p)=\int d q(t) d p(t) X(q(t), p(t)) \rho^{\prime}
$$

where $\rho^{\prime}$ is the same probability distribution expressed in terms of the current coordinates and momenta $q(t), p(t)$. The Liouville theorem of classical mechanics $d p d q=d p(t) d q(t)$ allowed us to make that change of variables.

Constructed in this way, $\rho^{\prime}$ is not a stationary probability distribution. But we expect it to have some limit as $t \rightarrow \infty$ otherwise the system we study does not have stationary states. But even if such a limit exists, it does not automatically imply all the correlation functions will also have some well defined limit as $t \rightarrow \infty$. This phenomenon will be discussed later in this paper. Now it will be enough to say that the additional conditions $\rho^{\prime}$ should satisfy to give a stationary state for the system is called the kinetic equation. To uniquely find the probability distribution one must solve the kinetic equation. Thermodynamic equilibrium is often just one of many solutions. 
The main problem of the theory of turbulence is to solve the kinetic equation and to find the correlation functions, especially the two point correlation function $\left\langle a_{p}^{\dagger} a_{p}\right\rangle$ which gives the spectrum of the theory.

In this paper we are going to study the kinetic equation for the system (1.1) and develop a diagrammatic technique to represent the infinite series in powers of $\lambda$ we will have to deal with. This technique while somewhat similar to that of $\varphi^{4}$ theory will have many features not encountered in Field Theory before. It may be even viewed as a generalization of $\varphi^{4}$ technique since in the limit of thermodynamic equilibrium it reduces to the standard $\varphi^{4}$ theory.

Nevertheless we are going to find some specific cases, namely when the interaction coefficient $\lambda$ is just a product of the external momenta, when one can extract the infinite series of most divergent diagrams, just like in $\varphi^{4}$ theory in four dimensions, with the condition of the dimensionlessness of the interaction coefficient, of course. With some additional assumptions, in particular neglecting the IR divergencies, we shall make use of it to calculate the correction to the "classical" spectrum of the theory.

Technically there is no need to know the probability distribution to compute all the correlation functions of the theory (11.1) as discussed later in the paper. But we hope that the study of the probability distribution for (1.1) will allow us to understand better the behavior of the correlation function of the hydrodynamic turbulence where no reliable methods of finding the correlation functions exist. We shall discuss that in the end of the paper.

The method which was widely used to compute the correlation functions thus far is called the Wyld's technique (see refs. [3], 河). It is essentially a classical nonequilibrium diagrammatic technique, and it deals with the development of the correlation functions in time unlike the technique proposed in this paper which deals with the equal time correlation functions, as in statistical mechanics. Recently a paper [5] was published where the authors claimed they could in principle compute the kinetic equation up to any order of the interaction using the Wyld's technique. We think that while the kinetic equation obtained in this paper should be equivalent with that of [5], our method is easier for practical calculation. 


\section{The Probability Distribution}

According to the program outlined in the Introduction, first of all we need to study all possible integrals of motion for the system (1.1). Let us see how one can construct those integrals. We shall start by assigning the variables $a_{p}$ and $a_{p}^{\dagger}$ the initial values $a_{p}(t=0)=a_{p}^{0}, a_{p}^{\dagger}(t=0)=a_{p}^{\dagger 0}$. Then by solving the equations of motion one can find $a(t)$ and $a^{\dagger}(t)$ as functions of $a^{0}$ and $a^{\dagger 0}$ and time. By inverting those functions, one can find $a^{0}$ and $a^{\dagger 0}$ as functions of time, $a(t)$ and $a^{\dagger}(t)$. But by definition $a^{0}$ and $a^{\dagger 0}$ do not depend on time. So they are the integrals of motion. If we want to construct something out of them which can play the role of the density of waves, we should consider the linear combination

$$
F=\sum_{p} f_{p} a_{p}^{\dagger 0} a_{p}^{0}
$$

where $f_{p}$ are some arbitrary coefficients. After being expressed in terms of $a(t), a^{\dagger}(t)$ and $t$ it becomes a valid integral of motion. Its explicit time dependence can be eliminated by passing to the limit $t \rightarrow \infty$.

In order to find the explicit form for $F$ we need to solve the equations of motion. The Hamiltonian (1.1) allows us to solve them perturbatively and we can obtain $F$ in terms of a series in powers of $\lambda$. We can even avoid solving the equations of motion if we use the following procedure. One should look for $F$ in terms of a power series

$$
\begin{aligned}
F=\sum_{p} f_{p} a_{p}^{\dagger} a_{p}+ & \sum \Lambda_{p_{1} p_{2} p_{3} p_{4}} a_{p_{1}}^{\dagger} a_{p_{2}}^{\dagger} a_{p_{3}} a_{p_{4}}+ \\
& +\sum \Omega_{p_{1} p_{2} p_{3} p_{4} p_{5} p_{6}} a_{p_{1}}^{\dagger} a_{p_{2}}^{\dagger} a_{p_{3}}^{\dagger} a_{p_{4}} a_{p_{5}} a_{p_{6}}+\ldots
\end{aligned}
$$

Here $\Lambda$ and $\Omega$ are some still unknown functions. We impose the condition on $F$ that it is an integral of motion, or $\{H F\}=0,\{$ and $\}$ being the Poisson brackets. It allows us to find those functions to get

$$
\begin{gathered}
\Lambda_{p_{1} p_{2} p_{3} p_{4}}=\frac{f_{p_{1}}+f_{p_{2}}-f_{p_{3}}-f_{p_{4}}}{\omega_{p_{1}}+\omega_{p_{2}}-\omega_{p_{3}}-\omega_{p_{4}}-i \epsilon} \lambda_{p_{1} p_{2} p_{3} p_{4}} \\
\Omega_{p_{1} p_{2} p_{3} p_{4} p_{5} p_{6}}=4 \sum_{p_{7}} \frac{\left(\lambda_{p_{7} p_{1} p_{5} p_{6}} \Lambda_{p_{2} p_{3} p_{4} p_{7}}-\Lambda_{p_{7} p_{1} p_{5} p_{6}} \lambda_{p_{2} p_{3} p_{4} p_{7}}\right)}{\omega_{p_{1}}+\omega_{p_{2}}+\omega_{p_{3}}-\omega_{p_{4}}-\omega_{p_{5}}-\omega_{p_{6}}-2 i \epsilon} .
\end{gathered}
$$

We can in principle find recursively all the terms in the series (2.2), one after another.

A few words must be said about $\epsilon$ 's which appear in the denominators. Technically, when we compute Poisson brackets no $\epsilon$ 's appear. But we must introduce them to avoid 
the poles in (2.3) and (2.4) in order for the sums in (2.2) to make sense. This procedure is legitimate only if we deal with the infinite number of degrees of freedom, that is the sums in (2.2) are really the integrals. If we had dealt with finite dimensional system, we would have obtained unavoidable zeros in the denominators of 2.2 and the integrals of motion we discuss would not have existed. This is a very important observation.

Another way to see why $\epsilon$ must appear is to use the original procedure of finding $F$. One would discover that the function $F$ depends on the expressions like $(1-\exp (-i \omega t)) / \omega$ and the only way to pass to the limit $t \rightarrow \infty$ is to assume that $\omega$ has an infinitesimal imaginary part. The last procedure ensures the signs of $\epsilon$ 's are correct.

It may seem somewhat strange that we paid attention to writing $2 i \epsilon$ in the denominator of $\Omega$ as $1 /(x-i \epsilon)=1 / x+i \pi \delta(x)$ regardless of the magnitude of $\epsilon$. However, later we shall see the cases when the magnitude of $\epsilon$ becomes important, in particular when we deal with the expressions like $\epsilon \delta(x) /(x-i \epsilon)$. To check the magnitude of $\epsilon$ of the denominator of $\Omega$, one should follow the finite time procedure described in the previous paragraph carefully. One would discover that the denominator of $\Omega$ came from summing up the denominators of two $\Lambda$ 's included in the definition of $\Omega$. If each of these $\Lambda$ 's has $-i \epsilon$ in its denominator, then there should be $-2 i \epsilon$ in the denominator of $\Omega$. This point will be clarified later in the paper.

The parameter $\epsilon$ plays the role of a regulator in the theory to be discussed and in this sense is analogous to the cutoff used in Quantum Field Theory ${ }^{\dagger}$. The function $F$ by itself is only an approximate integral of motion as long as $\epsilon$ is finite and we must pass to the limit $\epsilon \rightarrow 0$ in all our calculations.

The construction of the integrals of motion (2.2) represents a certain paradox. The system given by (1.1) is not necessarily integrable. Moreover, we are not interested in integrable systems. If the system were integrable, we could always make a change of variables to bring it to the form of noninteracting oscillators where no fluxes of energy could exist. Nevertheless we have just constructed the complete set of the integrals of motion for (1.1). Something must be wrong with those integrals, otherwise the system (1.1) would be integrable.

It is those poles in the denominators of (2.3) and (2.4) which are responsible for those integrals not to give rise to any integrability. They actually make the integrals (2.2) to be multi-valued functions which cannot be used to construct those tori the trajectories of

$\dagger$ It is a time cutoff. The momentum cutoff will also have to be imposed if needed. 
integrable systems lie on. But (2.2) can perfectly be used to play the role of probability distribution if one also introduces $\epsilon$ 's to regularize them. The finite time computation, as was discussed above, validates the introduction of $\epsilon$ 's in the form as in (2.3) and (2.4).

Now our statement is all the statistical states of the theory (1.1) can be described by the probability density given by

$$
\exp (-F)
$$

Even a thermodynamic equilibrium is a particular case of (2.5), with $f_{p}=\omega_{p}$ or $f_{p}=1$. All the correlation functions of the theory can be found using

$$
<X>\equiv \frac{1}{Z} \int \prod_{p} d a_{p} \prod_{p} d a_{p}^{\dagger} X \exp (-F)
$$

where $Z$ is a partition function

$$
Z=\int \prod_{p} d a_{p} \prod_{p} d a_{p}^{\dagger} \exp (-F)
$$

In a complete analogy with Quantum Field Theory, the role of the partition function in the denominator amounts just to removing "vacuum diagrams", or contractions totally inside the interaction, so we will usually omit it in our formulae while keeping that in mind.

We are going now to demonstrate how one can derive a well-known kinetic equation (in the first nonvanishing order of perturbation theory) using our probability distribution. The kinetic equation is just a statement that the derivative with respect to the time of the correlation function $<a_{p}^{\dagger} a_{p}>$ is zero. In other words,

$$
\begin{gathered}
<\frac{d\left(a_{p}^{\dagger} a_{p}\right)}{d t}>=<\left\{H, a_{p}^{\dagger} a_{p}\right\}>= \\
=-i \sum_{p_{1} p_{2} p_{3} p_{4}} \lambda_{p_{1} p_{2} p_{3} p_{4}}<a_{p_{1}}^{\dagger} a_{p_{2}}^{\dagger} a_{p_{3}} a_{p_{4}}>\left(\delta_{p p_{1}}+\delta_{p p_{2}}-\delta_{p p_{3}}-\delta_{p p_{4}}\right)=0
\end{gathered}
$$

Since the function $F$ is real, one can easily see from (2.8) that only the imaginary part of the correlation function $<a_{p_{1}}^{\dagger} a_{p_{2}}^{\dagger} a_{p_{3}} a_{p_{4}}>$ will contribute to the kinetic equation which can actually written down in the form

$$
-4 i \sum_{p_{2} p_{3} p_{4}} \lambda_{p_{2} p_{3} p_{4}} \operatorname{Im}<a_{p}^{\dagger} a_{p_{2}}^{\dagger} a_{p_{3}} a_{p_{4}}>=0
$$


Let us find the four point correlation function perturbatively. Its zero order value is equal to

$$
\begin{gathered}
<a_{p_{1}}^{\dagger} a_{p_{2}}^{\dagger} a_{p_{3}} a_{p_{4}}>\equiv \frac{1}{Z} \int \prod_{p} d a_{p} \prod_{p} d a_{p}^{\dagger} a_{p_{1}}^{\dagger} a_{p_{2}}^{\dagger} a_{p_{3}} a_{p_{4}} \exp \left(-\sum f_{p} a_{p}^{\dagger} a_{p}\right)= \\
=\frac{1}{f_{p_{1}} f_{p_{2}}}\left(\delta_{p_{1} p_{3}} \delta_{p_{2} p_{4}}+\delta_{p_{1} p_{4}} \delta_{p_{2} p_{3}}\right)
\end{gathered} .
$$

This expression is real so it will not contribute to the equation (2.9). Now the first order contribution to that function is obtained by expanding the exponent in powers of $\lambda$ and is given by

$$
-4 \lambda_{p_{1} p_{2} p_{3} p_{4}} \frac{f_{p_{3}}+f_{p_{4}}-f_{p_{1}}-f_{p_{2}}}{\omega_{p_{3}}+\omega_{p_{4}}-\omega_{p_{1}}-\omega_{p_{2}}-i \epsilon} \frac{1}{f_{p_{1}} f_{p_{2}} f_{p_{3}} f_{p_{4}}}+R
$$

where $R$ is a real expression coming from the contractions inside the interaction which is not interesting because it is real and also because it is zero in case if the interaction $\lambda$ conserves the momentum. Imaginary part of (2.10) is

$$
4 i \pi \lambda_{p_{1} p_{2} p_{3} p_{4}}\left(f_{p_{1}}+f_{p_{2}}-f_{p_{3}}-f_{p_{4}}\right) \delta\left(\omega_{p_{1}}+\omega_{p_{2}}-\omega_{p_{3}}-\omega_{p_{4}}\right) \frac{1}{f_{p_{1}} f_{p_{2}} f_{p_{3}} f_{p_{4}}}
$$

and after substituting it to (2.9) we arrive at the kinetic equation

$$
16 \pi \sum_{p_{2} p_{3} p_{4}} \lambda_{p p_{2} p_{3} p_{4}}^{2} \delta\left(\omega_{p}-\omega_{p_{2}}-\omega_{p_{3}}-\omega_{p_{4}}\right) \frac{f_{p}+f_{p_{2}}-f_{p_{3}}-f_{p_{4}}}{f_{p} f_{p_{2}} f_{p_{3}} f_{p_{4}}}=0 .
$$

One can easily recognize the standard kinetic equation of ref. [1] if one substitutes with the same accuracy $<a_{p}^{\dagger} a_{p}>=1 / f_{p}$.

\section{The Anomaly}

As it has already been mentioned in the Introduction, it is highly nontrivial that the kinetic equation we obtained is not identically zero. How can a stationary probability distribution give rise to a correlation function depending on time? More precisely we can use the following argument. For any quantity $X$

$$
<\frac{d X}{d t}>=<\{H X\}>=\int\{H X\} \exp (-F)=\int\{H F\} X \exp (-F) \equiv 0
$$

where we took the integral by parts and used that $F$ is the integral of motion, that is $\{H F\}=0$. But it contradicts our computations. The resolution of this paradox lies in 
the fact that $F$ is the integral of motion only in the limit $\epsilon \rightarrow 0$. If we compute $\{H F\}$ we shall discover that it is of the order of $\epsilon$. But then, when computing the integral $\int\{H F\} X \exp (-F)$, we shall find that it in itself is of the order of $1 / \epsilon$. Those epsilons cancel and we can safely pass to the limit $\epsilon \rightarrow 0$ to get the finite answer for the kinetic equation. The effect is completely analogous to the anomaly of Quantum Field Theory. By imposing the cutoff $\epsilon$ on the theory we violate the conservation of $F$ and it is never fully restored after removing the cutoff ${ }^{\dagger}$.

It is instructive to rederive the kinetic equation in this fashion. The Poisson bracket $\{H F\}$ can be recursively computed to give

$$
\{H F\}=-\epsilon \Lambda_{p_{1} p_{2} p_{3} p_{4}} a_{p_{1}}^{\dagger} a_{p_{2}}^{\dagger} a_{p_{3}} a_{p_{4}}-2 \epsilon \Omega_{p_{1} p_{2} p_{3} p_{4} p_{5} p_{6}} a_{p_{1}}^{\dagger} a_{p_{2}}^{\dagger} a_{p_{3}}^{\dagger} a_{p_{4}} a_{p_{5}} a_{p_{6}}-\ldots
$$

Then

$$
<\frac{d\left(a_{p}^{\dagger} a_{p}\right)}{d t}>=\int a_{p}^{\dagger} a_{p}\{H F\} \exp (-F) .
$$

The first order contribution to this expression is

$$
\begin{aligned}
\int \sum_{p_{1} p_{2} p_{3} p_{4}} a_{p}^{\dagger} a_{p} a_{p_{1}}^{\dagger} a_{p_{2}}^{\dagger} a_{p_{3}} a_{p_{4}} \exp \left(\sum_{k}-f_{k} a_{k}^{\dagger} a_{k}\right) \\
\frac{f_{p_{1}}+f_{p_{2}}-f_{p_{3}}-f_{p_{4}}}{\omega_{p_{1}}+\omega_{p_{2}}-\omega_{p_{3}}-\omega_{p_{4}}-i \epsilon} \lambda_{p_{1} p_{2} p_{3} p_{4}} \epsilon=0 .
\end{aligned}
$$

It is equal to zero even with $\epsilon>0$ as the contractions will enforce $f_{p_{1}}+f_{p_{2}}-f_{p_{3}}-f_{p_{4}}=0$.

The second order contribution to (3.2) is given by

$$
\begin{gathered}
\int a_{p}^{\dagger} a_{p}\left(-2 \epsilon \Omega_{p_{1} p_{2} p_{3} p_{4} p_{5} p_{6}} a_{p_{1}}^{\dagger} a_{p_{2}}^{\dagger} a_{p_{3}}^{\dagger} a_{p_{4}} a_{p_{5}} a_{p_{6}}+\right. \\
\left.+\epsilon \Lambda_{p_{1} p_{2} p_{3} p_{4}} \Lambda_{p_{5} p_{6} p_{7} p_{8}} a_{p_{1}}^{\dagger} a_{p_{2}}^{\dagger} a_{p_{p_{3}}} a_{p_{4}} a_{p_{5}}^{\dagger} a_{p_{6}}^{\dagger} a_{p_{7}} a_{p_{8}}\right) \exp \left(\sum_{k}-f_{k} a_{k}^{\dagger} a_{k}\right) .
\end{gathered}
$$

The integral involving $\Omega$ will be of the order of $1 / \epsilon$ as the contractions will ensure the denominator in (2.4) to be equal to $-2 i \epsilon$. After rather long and tedious computations (which actually become quite simple with some practice) we obtain

$$
2 \epsilon \int a_{p}^{\dagger} a_{p} \Omega a^{\dagger} a^{\dagger} a^{\dagger} a a a \exp \left(-\sum f_{k} a_{k}^{\dagger} a_{k}\right)=Q_{p}+P_{p}-K_{p}
$$

\footnotetext{
$\dagger$ I am indebted to I. Kogan for pointing out this correspondence.
} 
where

$$
\begin{gathered}
P_{p}=\sum_{p_{1} p_{2} p_{3} p_{4}} \frac{4 \pi}{f_{p} f_{p_{1}} f_{p_{2}} f_{p_{3}} f_{p_{4}}} \lambda_{p_{1} p_{2} p_{3} p_{4}}^{2} \delta\left(\omega_{p_{1}}+\omega_{p_{2}}-\omega_{p_{3}}-\omega_{p_{4}}\right) \\
\left(f_{p_{1}}+f_{p_{2}}-f_{p_{3}}-f_{p_{4}}\right)^{2}\left[\delta_{p p_{1}}+\delta_{p p_{2}}+\delta_{p p_{3}}+\delta_{p p_{4}}\right], \\
Q_{p}=\sum_{p_{1} p_{2} p_{3} p_{4}} \frac{4 \pi}{f_{p} f_{p_{1}} f_{p_{2}} f_{p_{3}} f_{p_{4}}} \lambda_{p_{1} p_{2} p_{3} p_{4}}^{2} \\
\delta\left(\omega_{p_{1}}+\omega_{p_{2}}-\omega_{p_{3}}-\omega_{p_{4}}\right)\left(f_{p_{1}}+f_{p_{2}}-f_{p_{3}}-f_{p_{4}}\right)^{2},
\end{gathered}
$$

and

$$
K_{p}=\sum_{p_{2} p_{3} p_{4}} \frac{16 \pi}{f_{p} f_{p_{2}} f_{p_{3}} f_{p_{4}}} \lambda_{p p_{2} p_{3} p_{4}}^{2}\left(f_{p}+f_{2}-f_{3}-f_{4}\right) \delta\left(\omega_{p}+\omega_{p_{2}}-\omega_{p_{3}}-\omega_{p_{4}}\right)
$$

The term $Q_{p}$ in (3.5) came from contracting of $a_{p}$ with $a_{p}^{\dagger}$ while the rest of the terms came from intercontracting those operators with the interaction. We recognize the kinetic equation (2.11) in $K_{p}=0$ while the term $P_{p}$ has the meaning of the entropy of waves ${ }^{\dagger}$.

As for the integral involving $\Lambda^{2}$, its divergent part is determined by the formula

$$
\frac{1}{(x+i \epsilon)(x-i \epsilon)} \rightarrow \frac{\pi}{\epsilon} \delta(x), \quad \epsilon \rightarrow 0
$$

to give

$$
\epsilon \int a_{p}^{\dagger} a_{p} \Lambda a^{\dagger} a^{\dagger} a a \Lambda a^{\dagger} a^{\dagger} a a \exp \left(-f a^{\dagger} a\right)=Q_{p}+P_{p}
$$

The difference between (3.5) and (3.9) gives us exactly the kinetic equation (2.11) as we expected.

However the calculations we just completed imply more than just checking the validity of integrating by parts. We can use the above formulae to calculate the corrections to the wave density $<a_{p}^{\dagger} a_{p}>$. We obtain

$$
<a_{p}^{\dagger} a_{p}>=\frac{1}{f_{p}}+\int a_{p}^{\dagger} a_{p}\left(-\Omega a^{\dagger} a^{\dagger} a^{\dagger} a a a+\frac{\Lambda \Lambda}{2} a^{\dagger} a^{\dagger} a a a^{\dagger} a^{\dagger} a a\right) \exp \left(-f a^{\dagger} a\right) .
$$

These integrals have already been computed. The only difference with the integrals in (3.5) and (3.9) is the absence of the "vacuum diagrams" or $Q_{p}$, and of course $\epsilon$ will not be canceled. That makes a crucial difference with (3.4). Not only the expressions we shall

$\dagger$ See ref. [1] for details on the entropy definition. 
obtain will be divergent with $\epsilon \rightarrow 0$, but also we need to extract the final parts from them, for example by using

$$
\frac{1}{(x+i \epsilon)(x-i \epsilon)} \rightarrow \frac{\pi}{\epsilon} \delta(x)-\frac{1}{x} \frac{\partial}{\partial x}+o(\epsilon)
$$

We shall discuss the formulae like (3.11) later in more details. Now we just note that in addition to the divergent with $\epsilon \rightarrow 0$ part, there will be a finite part which we shall not calculate here, leaving it to the discussion of the self energy corrections later in this paper.

The answer will be

$$
<a_{p}^{\dagger} a_{p}>=\frac{1}{f_{p}}-\frac{1}{2 \epsilon}\left(P_{p}-K_{p}\right)+\frac{1}{2 \epsilon} P_{p}+\frac{1}{\epsilon} O(\epsilon)=\frac{1}{f_{p}}+\frac{1}{2 \epsilon} K_{p}+\text { finite part }
$$

We see that the second order contribution to the wave density is clearly divergent and it should not be strange. The $\epsilon$ regularization actually plays the role of cutting off the times greater than $1 / \epsilon$ and if something is equal to $1 / \epsilon$, that means it is divergent with time going to infinity which can happen to the wave density if the kinetic equation is not satisfied. However if it is satisfied, we would rather expect no divergencies in (3.12). So it is reasonable to believe that the divergent higher order corrections to (3.12) will sum up to be $1 / \epsilon$ multiplied by the exact kinetic equation (2.9). As long as the kinetic equation is satisfied, we can neglect those corrections.

\section{Lowest Order Corrections to the Kinetic Equation}

With the experience of calculating different correlation functions we acquired in the previous section, we are ready to start computing the corrections to the kinetic equation. All we need to do is to take (2.9) and compute the imaginary part of the four point correlation function order by order by expanding $\exp (-F)$. The calculations are messy, but pretty straightforward. In this section we are are going to discuss some of the lowest order corrections to the equation (2.11), the ones which are not the corrections to the propagators, just to get acquainted with them. They consist of six terms. We begin with considering the first three of them. They are

$$
\begin{gathered}
\sum_{p_{1} p_{2} p_{3} p_{4} p_{5} p_{6}} 8 \pi \lambda_{p_{1} p_{2} p_{3} p_{4}} \lambda_{p_{3} p_{4} p_{5} p_{6}} \lambda_{p_{5} p_{6} p_{1} p_{2}}\left(\frac{f_{p_{1}}+f_{p_{2}}-f_{p_{3}}-f_{p_{4}}}{f_{p_{1}} f_{p_{2}} f_{p_{3}} f_{p_{4}}} \times\right. \\
\delta\left(\omega_{p_{1}}+\omega_{p_{2}}-\omega_{p_{3}}-\omega_{p_{4}}\right) \frac{f_{p_{5}}+f_{p_{6}}}{f_{p_{5}} f_{p_{6}}\left(\omega_{p_{5}}+\omega_{p_{6}}-\omega_{p_{3}}-\omega_{p_{4}}\right)}+ \\
+(3,4) \leftrightarrow(5,6)+(1,2) \leftrightarrow(5,6))\left[\delta_{p p_{1}}+\delta_{p p_{2}}-\delta_{p p_{3}}-\delta_{p p_{4}}\right]
\end{gathered}
$$

$(1,2) \leftrightarrow(5,6)$ means that this expression differs from the explicitly written one by exchanging the indeces. 


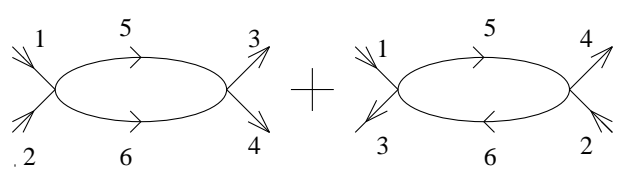

Fig. 1: Some of the lowest order corrections to the four point function

It is easy to represent the way how $\lambda$ 's are connected with each other by a simple diagram borrowed from the $\varphi^{4}$ theory. The only feature we have to add is the little arrows on each line representing whether the line goes into the vertex or from the vertex. Since $\lambda_{p_{1} p_{2} p_{3} p_{4}}$ is included in any correction (it comes from the exact equation (2.9) rather than from the perturbative expansion), there is no need to represent it. The two remainings $\lambda$ 's are represented by the first of the diagrams on fig. 1 .

As for the coefficient in front of the $\lambda^{\prime}$ 's, it doesn't resemble anything in $\varphi^{4}$ theory. The only familiar feature it retained from that theory is the product of all the propagators $1 /\left(f_{p_{1}} f_{p_{2}} f_{p_{3}} f_{p_{4}} f_{p_{5}} f_{p_{6}}\right)$. All the rest came from the interaction coefficients (2.4) and (2.3) which are very nontrivial. The next section of this paper will be devoted to their analysis. Now we shall only note the fact that the first term in (4.1) coincides with the kinetic equation (2.11) itself multiplied by

$$
\frac{f_{p_{5}}+f_{p_{6}}}{f_{p_{5}} f_{p_{6}}\left(\omega_{p_{5}}+\omega_{p_{6}}-\omega_{p_{3}}-\omega_{p_{4}}\right)} .
$$

The two intermediate lines in the diagram should be represented by (4.2). The second and the third terms in (4.1) are constructed from the first one, so we don't need to discuss them separately.

The expression (4.2) has a simple physical meaning. The scattering represented by the diagram we consider can happen in two ways. Either the waves 1 and 2 scatter to 5 and 6 , and then 5 and 6 scatter to 3 and 4, or the waves 5 and 6 first scatter to 3 and 4 , and only then 1 and 2 scatter to 5 and 6 . The first amplitude should be proportional to $\left(n_{5}+1\right)\left(n_{6}+1\right)$ where $n=1 / f$ is a wave density while the second to $n_{5} n_{6}$. The "energy denominators", represented by the $\omega$ 's which are of the same origin as the similar denominators in quantum mechanical perturbation theory, will have opposite signs in these two cases so the amplitude will be proportional to $\left(n_{5}+1\right)\left(n_{6}+1\right)-n_{5} n_{6} \approx n_{5}+n_{6}$, that is exactly (4.2). 
The three terms corresponding to the second diagram from fig. 1 can be computed to be

$$
\begin{gathered}
\sum_{p_{2} p_{3} p_{4} p_{5} p_{6}} 32 \pi \lambda_{p_{1} p_{2} p_{3} p_{4}} \lambda_{p_{4} p_{5} p_{2} p_{6}} \lambda_{p_{6} p_{3} p_{1} p_{5}}\left(\frac{f_{p_{1}}+f_{p_{2}}-f_{p_{3}}-f_{p_{4}}}{f_{p_{1}} f_{p_{2}} f_{p_{3}} f_{p_{4}}} \times\right. \\
\delta\left(\omega_{p_{1}}+\omega_{p_{2}}-\omega_{p_{3}}-\omega_{p_{4}}\right) \frac{f_{p_{5}}-f_{p_{6}}}{f_{p_{5}} f_{p_{6}}\left(\omega_{p_{5}}+\omega_{p_{4}}-\omega_{p_{6}}-\omega_{p_{2}}\right)}+ \\
+(3,1) \leftrightarrow(5,6)+(2,4) \leftrightarrow(5,6))\left[\delta_{p p_{1}}+\delta_{p p_{2}}-\delta_{p_{3}}-\delta_{p p_{4}}\right]
\end{gathered}
$$

Here the intermediate waves propagate in the opposite directions, so the amplitude is proportional to $\left(n_{5}+1\right) n_{6}-n_{5}\left(n_{6}+1\right) \approx n_{6}-n_{5}$.

\section{Diagrammatic Technique}

We can proceed with calculating higher order diagrams in the same fashion. But first we shall consider another approach of calculating the corrections to the four point correlation function, the one which doesn't require the knowledge of the probability distribution. While it gives the same results, it is sometimes simpler to use for practical computations. This approach is based on the idea that the correlation functions should not depend on time. For example, if we compute the time derivative of the four point correlation function, we obtain

$$
\begin{aligned}
<\left\{H, a_{1}^{\dagger} a_{2}^{\dagger} a_{3} a_{4}\right\} & >=i<a_{1}^{\dagger} a_{2}^{\dagger} a_{3} a_{4}>\left(\omega_{1}+\omega_{2}-\omega_{3}-\omega_{4}\right)+ \\
+ & \sum_{p_{1} p_{2} p_{3} p_{4}}<\left\{\lambda_{p_{1} p_{2} p_{3} p_{4}} a_{p_{1}}^{\dagger} a_{p_{2}}^{\dagger} a_{p_{3}} a_{p_{4}}, a_{1}^{\dagger} a_{2}^{\dagger} a_{3} a_{4}\right\}>=0
\end{aligned}
$$

which expresses the four point correlation function in terms of the six point one. Clearly, the zero order value of the six point correlation function will give us the first order value to the four point one and so on. Quite analogously, to obtain the first order correction to the six point correlation function, we write that its derivative is zero to express it in terms of eight point correlation function and so on. In order to avoid the poles while using (5.1) we replace $\sum \omega$ by $\sum \omega+i \epsilon$. The sign of the $\epsilon$ can be checked to be + by computing everything at finite time $^{\dagger}$.

$\dagger$ That means by solving the equation for the time derivative of the four point function and choosing the imaginary part for the $\omega$ to be such that there's a finite limit at $t \rightarrow \infty$. 
Our aim is to find the perturbation series for the four point correlation function, as clear from the exact kinetic equation (2.9). From the above consideration we see that the n-th order correction will be of the form

$$
i^{n}<a_{1}^{\dagger} a_{2}^{\dagger} a_{3} a_{4}>_{n}=<\frac{\left\{H_{i}, \frac{\left\{H_{i}, \ldots, \frac{\left\{H_{i}, a_{1}^{\dagger} a_{2}^{\dagger} a_{3} a_{4}\right\}}{\sum(\omega)+i \epsilon}\right\}}{\sum(\omega)+(n-1) i \epsilon}\right\}}{\sum(\omega)+n i \epsilon}>_{0}
$$

where $H_{i}$ is the interaction part of the hamiltonian (1.1),

$$
H_{i}=\sum_{p_{1} p_{2} p_{3} p_{4}} \lambda_{p_{1} p_{2} p_{3} p_{4}} a_{p_{1}}^{\dagger} a_{p_{2}}^{\dagger} a_{p_{3}} a_{p_{4}},
$$

and we retain just the zero order value for the correlation function on the right hand side of (5.2).

Let us see how (5.2) works. First we compute the Poisson bracket

$$
\left\{H_{i}, a_{1}^{\dagger} a_{2}^{\dagger} a_{3} a_{4}\right\}=i \sum \lambda_{p_{1} p_{2} p_{3} 1} a_{p_{1}}^{\dagger} a_{p_{2}}^{\dagger} a_{p_{3}} a_{2}^{\dagger} a_{3} a_{4}+\ldots
$$

We represent the product $a_{1}^{\dagger} a_{2}^{\dagger} a_{3} a_{4}$ by a vertex with the incoming lines 1 and 2 and the outgoing lines 3 and 4 . Taking the Poisson bracket means attaching another vertex to the original one with the lines $p_{1}, p_{2}, p_{3}$, and 1 , the last line going to the first vertex. Then, according to (5.2) we divide all we obtained by $\omega_{1}+\omega_{2}-\omega_{3}-\omega_{4}+i \epsilon$. Graphically, it is just the sum and difference of $\omega$ 's corresponding to the incoming and the outgoing lines of the first vertex. Then we continue the procedure. Taking the Poisson bracket again means attaching another vertex to the diagram we are building, and then we need to divide by the sum and difference of $\omega$ 's corresponding to the first and the second vertex taken together. At last, when we arrive at the $n$th order picture, which is called the tree diagram, we need to compute the Gaussian correlation function, that means to close each outgoing external line of the tree with the incoming line and multiplying the expression by the product of the propagators $1 / f$ for each Gaussian contraction. Simultaneously our picture transforms itself into something resembling the standard $\varphi^{4}$ theory diagram. The distinctive feature is the arrows on the lines (to distinguish between the incoming and outgoing lines) as we already saw in the previous section. Also, if we do everything honestly, we need to close the external lines 1, 2, 3, and 4 into one additional vertex, but that makes the drawing rather clumsy, so we shall be using the standard diagrams like those shown on fig. 1. 
In short, we see that the right hand side of (5.2) is constructed by connecting $\lambda$ 's from different $H_{i}$ together by means of the Poisson brackets to form a tree diagram and multiplying $\sum \omega$ in the denominator along the way, then by pairing all $a$ with $a^{\dagger}$ to form a real diagram writing the propagators $1 / f$ for each line which was formed by pairing and not by a Poisson bracket connections. The symbol $\sum \omega$ means first we take the sum of $\pm \omega$ 's belonging to the incoming or outgoing lines of the first vertex, then we take the sum of $\pm \omega$ belonging to the incoming or outgoing lines of the first and the second vertex along the tree "trunk" combined and so on.

We can reverse the procedure to say that first we should draw a $\varphi^{4}$ diagram, then cut some of its lines to turn it into a tree diagram and write down the product of $\sum \omega$ taken along the tree trunk. Then we multiply what we got by the product of $f$ taken alone the trunk and divide it by the product of all $f$ 's. And the last step should be summing over all different trees which can be curled up to form the same diagram. Each tree should have a direction along which we go and each trunk line then has a sign depending on whether this line has an arrow alone or opposite to that general direction. The sign of the diagram is the product of the signs of the trunk lines. It follows from the property of the Poisson bracket to have opposite signs in case when $a$ is connected to $a^{\dagger}$ or vice versa.

We can go a little bit further in the formulation of our rules of reading diagrams. Namely if the tree have many branches, there are many ways we can sum up the $\omega$ 's. We can go along the first branch of the tree, then the second, or first along the second, then the first, or we can go up half of the first branch, then along the second, and then finish off the first one. And we need to take the sum of all the expressions involving w's we obtain in this way.

To compute this sum is a difficult job, but fortunately the expressions conspire in such a way as to give rise to the formula which can be written down using the Feynman rules given below which work for both the trees with branches and without, and so they give the general way of computing any diagram without doing the actual computation.

\section{The Feynman rules.}

In order to write down the expression corresponding to a given diagram, we need:

1) To write down the product of $\lambda$ 's corresponding to the vertices of the diagram, just like in $\varphi^{4}$ theory.

2) To write down the product of all the propagators $1 / f$ corresponding to each line of the diagram, again just like in $\varphi^{4}$ theory. 
3) And to compute a special prefactor. First we need to make up a list of all possible expressions $\sum \omega$ taken around each vertex of the diagram and around each combination of vertices. Then we need to cut some of the lines of the diagram to convert it into a tree diagram. The tree diagram by definition always starts from the external line while the external lines are thought as being connected together by some "external" vertex. The expression corresponding to the tree will be the product of all $f$ 's taken along the trunk and the product of as many $1 /\left(\sum \omega\right)$ taken from the list we made up as there are $f$ 's, each of the $\sum \omega$ having one and only one particular omega belonging to one of the trunk lines. One can show there is one and only one subset of $\sum \omega$ with this property in the list we prepared. After doing that, we should replace all $\sum \omega$ by $\sum(\omega)-i n \epsilon$ where $n$ is the number of vertices we combined together to write down its $\sum \omega . n$ appears in front of $\epsilon$ since in combining together $\sum(\omega)-i \epsilon$ from adjacent vertices we have to sum their $\epsilon$ as well. $^{\dagger}$ The last step here is summing up over all possible trees keeping in mind that the trees are taken with plus or minus sign depending on their intrinsic signs as was explained above.

4) The analytical expression corresponding to a given diagram is the product of what we obtained in steps 1), 2), and 3).

We would like to remark here that even though we obtained our rules by the method given in the beginning of this section, one can check in every particular case that the expansion of the probability density $\exp (-F)$ will give the same result. It is clearly so as one can show the equations like (5.1) are always satisfied if the correlation functions are computed with the help of our probability density. Even though the probability distribution possesses not only the four point vertices but also six and higher order vertices, they in fact conspire to give only the $\varphi^{4}$ theory diagrams as the higher order vertices are the products of the four point ones as is clear from (2.3), (2.4), etc. If one tries to rigorously prove the Feynman rules by expanding the probability density, one obtains the expressions which, though much more complicated than those given above, reduce to those simpler expressions after some tedious algebraic computations. It actually looks like a miracle that so many terms cancel in this procedure. To prove the algebraic identities appearing in this

$\dagger$ We write here $-i \epsilon$ instead of $i \epsilon$ because we formulate these rules as computed in (2.2) representation. The reader may easily check it by comparing the $\sum \omega$ given in the rules with the previous $\sum \omega$ which are computed together with the external vertex. 
way is probably a very interesting but rather difficult task which we have not accomplished but which is not needed for our purposes.

Let us compute the expression for the first diagram from the fig. 1 using these rules to see how they work in real life. The first tree we choose goes along the lines $(1,5)$. The expression it corresponds to is

$$
\frac{f_{1} f_{5}}{\left(\omega_{1}+\omega_{2}-\omega_{3}-\omega_{4}-2 i \epsilon\right)\left(\omega_{5}+\omega_{6}-\omega_{3}-\omega_{4}-i \epsilon\right)}
$$

We have used a shorthand notation here $f_{n} \equiv f_{p_{n}}$.

Then there is another tree going along the lines $(1,6)$. Its expression differs from (5.3) only by replacing $f_{5}$ by $f_{6}$. So we can sum them up together to obtain

$$
\frac{f_{1}\left(f_{5}+f_{6}\right)}{\left(\omega_{1}+\omega_{2}-\omega_{3}-\omega_{4}-2 i \epsilon\right)\left(\omega_{5}+\omega_{6}-\omega_{3}-\omega_{4}-i \epsilon\right)}
$$

This is a part of a general rule. If there's a minimal loop consisting of the lines $n$ and $m$ going between two vertices, then we always have the factor of the form

$$
\frac{f_{n}+f_{m}}{\omega_{n}+\omega_{m}-\sum \omega}
$$

if the arrows of $n$ and $m$ are in the same direction and

$$
\frac{f_{n}-f_{m}}{\omega_{n}-\omega_{m}-\sum \omega}
$$

if they are in the opposite direction plus some other contributions which can appear if the tree trunk can go through both ends of the loop we consider without going along the lines $n$ or $m$.

Then there are trees going through $(2,5)$ or $(2,6)$. Their expressions are also almost the same as (5.3) with the replacement $f_{1} \leftrightarrow f_{2}$. We sum them up to obtain

$$
\frac{\left(f_{1}+f_{2}\right)\left(f_{5}+f_{6}\right)}{\left(\omega_{1}+\omega_{2}-\omega_{3}-\omega_{4}-2 i \epsilon\right)\left(\omega_{5}+\omega_{6}-\omega_{3}-\omega_{4}-i \epsilon\right)}
$$

Other trees without branches are $(3,5),(3,6),(4,5)$, and $(4,6)$. Their sum gives

$$
\frac{\left(f_{3}+f_{4}\right)\left(f_{5}+f_{6}\right)}{\left(\omega_{1}+\omega_{2}-\omega_{3}-\omega_{4}-2 i \epsilon\right)\left(\omega_{1}+\omega_{2}-\omega_{5}-\omega_{6}-i \epsilon\right)}
$$

The remaining trees are with branches. These are $(1,3),(1,4),(2,3),(2,4)$. Their expression can also be found using the Feynman rules. For example, $(1,3)$ gives

$$
-\frac{f_{1} f_{3}}{\left(\omega_{1}+\omega_{2}-\omega_{5}-\omega_{6}-i \epsilon\right)\left(\omega_{5}+\omega_{6}-\omega_{3}-\omega_{4}-i \epsilon\right)}
$$


while all four trees can be summed up to give

$$
-\frac{\left(f_{1}+f_{2}\right)\left(f_{3}+f_{4}\right)}{\left(\omega_{1}+\omega_{2}-\omega_{5}-\omega_{6}-i \epsilon\right)\left(\omega_{5}+\omega_{6}-\omega_{3}-\omega_{4}-i \epsilon\right)} .
$$

The minus sign appeared here as one of the lines of the tree trunks here goes the opposite way to the arrow of that line.

The total expression for the diagram is the sum of (5.7), (5.8), and (5.10). If we want to compute the correction to the kinetic equation, we need to take its imaginary part. We obtain three terms proportional to the three possible delta functions. For example, the term with $\delta\left(\omega_{3}+\omega_{4}-\omega_{1}-\omega_{2}\right)$ comes from (5.7) and (5.8) and is proportional to

$$
\begin{gathered}
\left(\frac{\left(f_{1}+f_{2}\right)\left(f_{5}+f_{6}\right)}{\omega_{3}+\omega_{4}-\omega_{5}-\omega_{6}}+\frac{\left(f_{3}+f_{4}\right)\left(f_{5}+f_{6}\right)}{\omega_{5}+\omega_{6}-\omega_{1}-\omega_{2}}\right) \delta\left(\omega_{3}+\omega_{4}-\omega_{1}-\omega_{2}\right)= \\
=\frac{\left(f_{1}+f_{2}-f_{3}-f_{4}\right)\left(f_{5}+f_{6}\right)}{\omega_{3}+\omega_{4}-\omega_{5}-\omega_{6}} \delta\left(\omega_{3}+\omega_{4}-\omega_{1}-\omega_{2}\right)
\end{gathered}
$$

It coincides with the first term of (4.1) after multiplying it by the necessary $\lambda$ 's and by all the propagators $1 / f$.

There is an interesting observation applicable for the expressions we obtain after taking the imaginary part. The $\delta$ functions which appear as soon as we take the imaginary part are always multiplied by the corresponding sum of $f$, that is all of them appear in the form $\delta\left(\sum \omega\right) \sum f$. It can be proved by analyzing the rules we discussed or, more easily, by noting that $f=\omega$ should always be an exact solution of the kinetic equation (2.9) and $\delta\left(\sum \omega\right) \sum \omega \equiv 0$ enforces that last statement.

As we start to compute higher order diagrams, additional feature we shall discover after taking the imaginary part is the appearance of the products of the odd number of $\delta$-functions in them.

A very important property of the diagrams of almost any field theory which our diagrams are missing is the "block summing" property. Our rules are such that adding a block inside one diagram is totally different from having this block inside another one. This poses a major difficulty in dealing with our technique. 

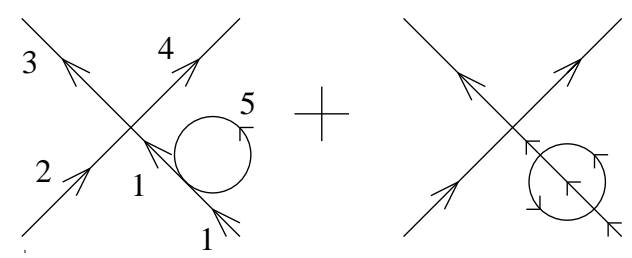

Fig. 2: Corrections to the propagators

Now, we immediately ran into new difficulties as soon as we consider self energy corrections diagrams like those shown on fig. 2. Such diagrams have at least two lines with the same momentum (because of the momentum conservation at vertices) and, by using our rules, we arrive at divergent expressions of the form

$$
\frac{1}{\omega-\omega+i \epsilon}=\frac{1}{i \epsilon}
$$

(For example, the first diagram of fig. 2 has a vertex with a tadpole loop growing from it and its $\sum(\omega)$ is cleary zero.) It is in fact in the expressions of this kind that the coefficients in front of $\epsilon$ become important.

The appearance of the divergent expressions is nothing new; we already dealt with them in the section 2 to discover that they are actually zero by virtue of the kinetic equation. But there are still finite parts left. To compute them, we have to make use of the following important formulae:

$$
\begin{gathered}
\int d x \frac{f(x)}{x \pm i \epsilon}=\sum_{n=0}^{\infty} \frac{(\mp i \epsilon)^{n}}{n !} \int d x \frac{f^{(n)}(x)}{x \pm i 0} \\
\int d x \frac{f(x)}{(x \pm i \epsilon)^{n}}=\frac{1}{n !} \int d x \frac{f^{(n-1)}(x)}{x \pm i \epsilon}
\end{gathered}
$$

These formulae are correct if the integrals are convergent. If they are not, we have to include the boundary terms on the right hand side.

Let us use them to calculate the first of two diagrams of fig. 2. We shall discover that this diagram, after adding to it all its generalizations, including those with many tadpole loops on the external lines, give the correction to the frequency $\omega$ in the kinetic equation (2.11). Really, the first diagram of fig. 2 can be computed to give

$$
\lambda_{p_{1} p_{2} p_{3} p_{4}}^{2} \frac{f_{p_{1}}+f_{p_{2}}-f_{p_{3}}-f p_{4}}{f_{p_{1}} f_{p_{2}} f_{p_{3}} f_{p_{4}}} \delta^{\prime}\left(\omega_{p_{1}}+\omega_{p_{2}}-\omega_{p_{3}}-\omega_{p_{4}}\right) \frac{\lambda_{p_{1} p_{5} p_{1} p_{5}}}{f_{p_{5}}}
$$


$\delta^{\prime}$ appears as we have to use (5.12) and (5.13) in giving sense to the expressions we obtained using the rules discussed above while the convergence of the integrals in (2.11) allows us not to worry about the boundary terms.

The expression here, when combined with the kinetic equation (2.11), clearly gives the correction to the frequency in the equation (2.11)

$$
\omega_{p} \rightarrow \omega_{p}+\int \frac{\lambda_{p q p q}}{f_{q}} d q
$$

While (5.14) is just the first term of the expansion due to 5.15 we can easily prove that (5.15) can be obtained up to any order if one sums up the diagrams with all possible tadpole graphs on the external lines. However, more complex diagrams, like the second one from the fig. 2, can lead to the corrections which cannot be interpreted that easily.

We would like to conclude this section with saying that the technique described here is more general than the standard field theory approach of the $\varphi^{4}$ theory. In fact, if we take the limit of $f_{p} \rightarrow \omega_{p}$ we shall discover that the prefactor computed according to our rules goes into 1 and the whole expression turns into the standard $\varphi^{4}$ theory diagram. This should of course be expected as in this limit $F \rightarrow H$. So our Feynman rules should be treated as the "turbulent" generalization of the standard field theory rules. We believe many beautiful results are hidden in this new technique.

\section{Epsilon Expansion}

The kinetic equation (2.11) is in general very difficult to solve (that is, to find such $f_{p}$ that it is satisfied). However it has long been realized that if both $\lambda$ and $\omega$ are homogeneous functions of momenta, then we can solve that equation exactly. Namely, following ref. [1] we choose

$$
\omega_{p}=p^{\alpha}, \quad \lambda_{\vec{p}_{1} \vec{p}_{2} \vec{p}_{3} \vec{p}_{4}}=\lambda_{0}\left(p_{1} p_{2} p_{3} p_{4}\right)^{\frac{\beta}{4}} U\left(\vec{p}_{1}, \vec{p}_{2}, \vec{p}_{3}, \vec{p}_{4}\right) \delta\left(\vec{p}_{1}+\vec{p}_{2}-\vec{p}_{3}-\vec{p}_{4}\right)
$$

where $U$ is a function depending only on the ratio of lengths of the momenta and the angles between them and $\lambda_{0}$ is a small constant. The parameter $\alpha$ is called the energy spectrum dimension, while $\beta$ is the interaction dimension. Then the kinetic equation can be solved with the aid of the so-called Zakharov transformations, to give

$$
f_{p}=p^{\gamma}, \gamma=\frac{2}{3} \beta+d \text { or } \gamma=\frac{2}{3} \beta+d-\frac{\alpha}{3}
$$


$d$ being the number of space dimensions. The latter of the solution corresponds to the flux of the wave number while the former to the energy flux. All that has been known for a long time.

However the solution for $f_{p}$ we get in this way is just a first approximation to the full answer which should in fact be a series in powers of $\lambda_{0}$ obtained as a solution to the exact kinetic equation, with all the higher order corrections. The situation is reminiscent to that of the second order phase transition theory (on the theory of the second order phase transitions, see ref. [6] and references therein). The behavior of the perturbation series depends crucially on whether the physical dimension (measured in powers of momentum) of $\lambda_{0}$ is positive or negative. Let us denote that dimension by $-\kappa$. Then, just from the analysis of the dimensions, we see that the series for $f_{p}$ should look like

$$
f_{p}=\sum_{n=0}^{\infty} c_{n} \lambda_{0}^{n} p^{\gamma+n \kappa}
$$

$c_{n}$ being just some dimensionless coefficients. This is of course true only if the momentum cutoff does not appear explicitly in the series, that is all the integrals in the kinetic equation are convergent, but let us first examine this case. In general we are interested in the behavior of the spectrum $f_{p}$ at the distances much larger than the dissipation length. That means that we should actually take $p \rightarrow 0$ when analyzing (6.3) (like in the phase transition theory again). Now if $\kappa>0$ then we can just retain the first term in the series (6.3), while if $\kappa<0$, we can no longer do that. We must sum up the whole series and analytically continue it to the region where $p \rightarrow 0$. So, $\kappa$ plays the role of the $\epsilon=d-4$ parameter of the theory of the second order phase transitions.

The method of phase transition theory to sum up the 6.3)-like series for $\kappa$ a small negative number is called the " $\epsilon$ - expansion" (refs. [7] and [6]). We are going to apply this method to the theory we have here.

First let us find what $\kappa$ is equal to. It is clear the physical dimension of the free wave part of the Hamiltonian (1.1) and the interaction part should be the same which leads us to

$$
\alpha-\gamma=-\kappa+\beta-d+2(-d-\gamma)+4 d
$$

or

$$
\kappa=\beta+d-\gamma-\alpha
$$

We took into account that the dimension of the fields $a$ and $a^{\dagger}$ is $-\gamma / 2-d / 2$. 
Let us note that upon substitution of (6.2) into (6.4) we obtain

$$
\kappa=\frac{\beta}{3}-\alpha \text { or } \kappa=\frac{\beta}{3}-\frac{2}{3} \alpha
$$

which shows that actually $\kappa$ does not depend on the space dimensionality at all. This is different from what we usually have in the second order phase transition theory.

Each next correction to the kinetic equation has a higher power of $\lambda_{0}$. But the total physical dimension of all the terms should be the same. That means the total power of the momenta in each next term will be greater by $\kappa$ than that in the previous one. $\kappa>0$ may lead to the divergent integrals in the perturbation series. However the divergency of the theory we consider does not depend on the power of the integrand only. It also depends on the properties of the interaction coefficient $U$. If $U$ decreases exponentially when the ratio of the momenta becomes high, then all the integrals will be convergent. In this case the solution $f_{p}=p^{\gamma}$ is not modified at $p \rightarrow 0$ for all $\kappa \geq 0$ (and even exact at $\kappa=0$ ) while $\kappa<0$ is very difficult, if not impossible, to analyze. That is why, as was promised in the Introduction, we are going to consider the opposite case, $U=1$, when the properties of the interaction do not influence the convergence of the integrals at all ${ }^{\dagger}$.

Now $\kappa>0$ does lead to the divergent integrals. However it should not bother us as all the integrals are supposed to be cut off at some high momentum $\Lambda$ where the energy dissipation takes place. The value of these integrals will depend on the value of the cutoff only and they change the kinetic equation (2.11) only by multiplying it by a number without changing its form. The situation is different when $\kappa<0$. Then the integrals in the perturbation series are more and more convergent and we should compute the sum (6.3).

There is also the third possibility, namely $\kappa=0$. In this case we can expect logarithmically divergent integrals and the series (6.3) may turn into

$$
f_{p}=p^{\gamma} \sum_{n=0}^{\infty} c_{n} \lambda_{0}^{n} \log ^{n}\left(\frac{\Lambda}{p}\right)
$$

The standard approach of the $\epsilon$-expansion theory tells us that if we are able to separate the most divergent diagrams which contribution are of the order of $\lambda_{0}^{n} \log ^{n}(\Lambda / p)$ and sum

$\dagger$ We must admit that it is the intermediate case which is usually fulfilled in Nature, that is $U$ behaves as a power asymptotically, but we shall consider $U=1$ to simplify everything as much as we can. 
them up, we can obtain a true asymptotic behavior $f_{p}$ for large logarithms. Now if $\kappa<0$ we can apply the trick of replacing the logarithms in (6.5) by $p^{\kappa}$ which will give us a modified wave density spectrum. This is a program we are going to fulfill.

As was promised many times, we choose $U=1$ in the interaction, or

$$
\lambda_{\vec{p}_{1} \vec{p}_{2} \vec{p}_{3} \vec{p}_{4}}=\lambda_{0}\left(p_{1} p_{2} p_{3} p_{4}\right)^{\frac{\beta}{4}} \delta\left(\vec{p}_{1}+\vec{p}_{2}-\vec{p}_{3}-\vec{p}_{4}\right)
$$

Also there will be an additional condition $\alpha<1$ we will have to impose later for the purpose of simplifying the calculations and which is very natural for the theory described by (1.1) (see [1]).

Let us begin with analyzing the simplest bubble diagrams shown on fig. 1. The special form of the interaction (6.6) enables us to disentangle the momenta in it to bring it to the form

$$
-2 K\left(\vec{p}_{1}, \vec{p}_{2}, \vec{p}_{3}, \vec{p}_{4}\right) \int d p_{5} d p_{6} \frac{p_{5}^{\gamma}+p_{6}^{\gamma}}{p_{5}^{\gamma} p_{6}^{\gamma}\left(p_{5}^{\alpha}+p_{6}^{\alpha}-p_{3}^{\alpha}-p_{4}^{\alpha}\right)}\left(p_{5} p_{6}\right)^{\frac{\beta}{2}} \delta\left(\vec{p}_{3}+\vec{p}_{4}-\vec{p}_{5}-\vec{p}_{6}\right)
$$

where we denoted the kernel of kinetic equation by $K$,

$$
\begin{gathered}
K\left(\vec{p}_{1}, \vec{p}_{2}, \vec{p}_{3}, \vec{p}_{4}\right)=\frac{4 \pi}{f_{p_{1}} f_{p_{2}} f_{p_{3}} f_{p_{4}}} \lambda_{p_{1} p_{2} p_{3} p_{4}}^{2}\left(f_{p_{1}}+f_{p_{2}}-f_{p_{3}}-f_{p_{4}}\right) \\
\delta\left(\omega_{p_{1}}+\omega_{p_{2}}-\omega_{p_{3}}-\omega_{p_{4}}\right)
\end{gathered}
$$

and assumed the integrals over $p_{1}, p_{2}, p_{3}$ and $p_{4}$ will be computed later.

The integral in (6.7) will be exactly logarithmically divergent at large momenta for $\kappa=0$. Namely if $p_{5}$ and $p_{6}$ are very large we can safely assume $\vec{p}_{6}=-\vec{p}_{5}$ eliminating the $\delta$-function in this way and be left with just one integral giving the $\log (\Lambda)$ at its upper limit. What is in the lower limit is not important, it is enough for us to write

$$
\log \left(\frac{\Lambda}{u\left(\vec{p}_{3}, \vec{p}_{4}\right)}\right)
$$

where $u$ is some homogeneous function of the first order. The correction to the kinetic equation (2.11) we thus obtained is just

$$
\left.\sum_{p_{1} p_{2} p_{3} p_{4}}-2 K\left(p_{1}, p_{2}, p_{3}, p_{4}\right) \lambda_{0} s \log \left(\frac{\Lambda}{u\left(\vec{p}_{3}, \vec{p}_{4}\right)}\right)\right)\left(\delta_{p p_{1}}+\delta_{p p_{2}}-\delta_{p p_{3}}-\delta_{p p_{4}}\right)
$$

Combining the correction we just obtained with the kinetic equation itself we arrive at the modified kinetic equation

$$
\sum_{p_{1} p_{2} p_{3} p_{4}} K\left(p_{1}, p_{2}, p_{3}, p_{4}\right)\left(1-2 \lambda_{0} s \log \left(\frac{\Lambda}{u\left(\vec{p}_{3}, \vec{p}_{4}\right)}\right)\right)\left(\delta_{p p_{1}}+\delta_{p p_{2}}-\delta_{p p_{3}}-\delta_{p p_{4}}\right)
$$


s being a number coming from the angular integration.

It is not difficult to persuade ourselves that the sum of the contributions of the second and the third terms of (4.1) will be equal to (6.9). Really, it is evident that

$$
\begin{gathered}
\sum_{p_{1} p_{2} p_{5} p_{6} p_{3} p_{4}} 8 \pi \lambda_{p_{1} p_{2} p_{5} p_{6}} \lambda_{p_{3} p_{4} p_{5} p_{6}} \lambda_{p_{3} p_{4} p_{1} p_{2}} \times \\
\frac{\left(f_{p_{1}}+f_{p_{2}}-f_{p_{5}}-f_{p_{6}}\right) \delta\left(\omega_{p_{1}}+\omega_{p_{2}}-\omega_{p_{5}}-\omega_{p_{6}}\right)\left(f_{p_{3}}+f_{p_{4}}\right)}{f_{p_{1}} f_{p_{2}} f_{p_{3}} f_{p_{4}} f_{p_{5}} f_{p_{6}}\left(\omega_{p_{3}}+\omega_{p_{4}}-\omega_{p_{5}}-\omega_{p_{6}}\right)}\left[\delta_{p p_{3}}+\delta_{p p_{4}}\right]=0
\end{gathered}
$$

since the expression above is antisymmetric under the interchange of $p_{1}, p_{2}$ with $p_{5}, p_{6}$. On the other hand, the same expression with $\delta_{p p_{1}}+\delta_{p p_{2}}$ will give us one half of (6.9). A similar thing can be said about the third term of (4.1). Together all of them give us twice the (6.9).

Unfortunately, these are not the only divergencies associated with this diagram. It is clear the diagram is going to be divergent for $p_{5} \rightarrow 0$ or $p_{6} \rightarrow 0$. The degree of the divergence is just $\beta / 2-\gamma$, so it is $-\alpha / 2$ for the energy spectrum when, due to the condition $\kappa=0, \beta$ should be equal to $3 \alpha$ or logarithmic for the wave number spectrum when $\beta=2 \alpha$. This divergence will bring additional correction to the kinetic equation

$$
\frac{q^{\frac{\alpha}{2}}}{a^{\frac{\alpha}{2}}} \text { or } \log (q / a)
$$

where $a$ is the IR cutoff while $\mathrm{q}$ is the momentum of the order of the external momenta.

However, if $q$ is sufficiently small, we can neglect the IR term we just obtained in comparison with the UV one. We admit that this statement is rather vague. In fact, to have a satisfying proof, we need to sum up all the IR corrections corresponding to different diagrams. Most probably, there is some sort of condensate being formed like in the very well known case of Bose gas which will cancel the divergencies. But this has not been shown yet. We intend to devote another paper to the IR problem. Here we just assume we can neglect all the IR terms. This simplifies things a great deal as we can neglect the IR corrections to the frequency as well. The frequency correction (5.15) is IR divergent with the same power as the bubble diagram we considered. We shall neglect that correction as well while leaving the task of evaluating the physical effects it can lead to to another paper. Acting consistently, we also neglect all the self energy correction diagrams.

Now we consider the second diagram given by

$$
-2 K\left(\vec{p}_{1}, \vec{p}_{2}, \vec{p}_{3}, \vec{p}_{4}\right) \int d p_{5} d p_{6} \frac{p_{5}^{\gamma}-p_{6}^{\gamma}}{p_{5}^{\gamma} p_{6}^{\gamma}\left(p_{5}^{\alpha}-p_{6}^{\alpha}+p_{4}^{\alpha}-p_{2}^{\alpha}\right)}\left(p_{5} p_{6}\right)^{\frac{\beta}{2}} \delta\left(\vec{p}_{4}-\vec{p}_{2}+\vec{p}_{5}-\vec{p}_{6}\right)
$$

It turns out the condition $\alpha<1$ makes it convergent. Really, $\vec{p}_{5} \approx \vec{p}_{6}$ in this case, thus enforcing $p_{5}^{\alpha}-p_{6}^{\alpha}<<1$. The power of the integrand is then estimated to be $\gamma-1-2 \gamma+$ $\beta+d=-1+\alpha<0$. 


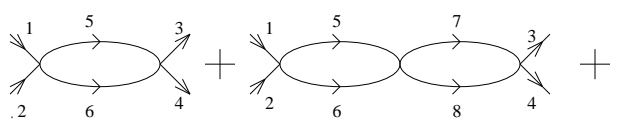

Fig. 3: The most important logarithmically divergent diagrams

The nontrivial statement about more complex diagrams is that when we go to the higher orders, only the diagrams shown on fig. 3 which are the direct generalization of (4.1) will give us the necessary power of the logarithm. The expressions corresponding to them can be easily established by using the rules of the previous section to be just the product of (4.2) for each loop. In addition to the logarithm to the power of $n$ each of such bubble diagram will bring a factor of $(-2 s)^{n}(n+1) \cdot n+1$ appears as the diagrams come with $2 n$ replicas different by the original ones by the interchange of indices which give us the same expression as the original ones divided by 2 .

We can simply sum up the whole series

$$
\sum_{n=0}^{\infty}(n+1)\left(-2 s \lambda_{0}\right)^{n} \log ^{n}\left(\frac{\Lambda}{u\left(\vec{p}_{3}, \vec{p}_{4}\right)}\right)=\frac{1}{\left[1+2 s \lambda_{0} \log \left(\frac{\Lambda}{u\left(\vec{p}_{3}, \vec{p}_{4}\right)}\right)\right]^{2}}
$$

So we obtained the full kinetic equation, with the most important higher order contributions summed up

$$
\begin{aligned}
\int d p_{1} d p_{2} d p_{3} d p_{4}\left(\delta_{p p_{1}}+\delta_{p p_{2}}-\delta_{p p_{3}}-\delta_{p p_{4}}\right) \\
\frac{\lambda_{p_{1} p_{2} p_{3} p_{4}}^{2}\left(f_{p_{1}}+f_{p_{2}}-f_{p_{3}}-f_{p_{4}}\right) \delta\left(\omega_{p_{1}}+\omega_{p_{2}}-\omega_{p_{3}}-\omega_{p_{4}}\right)}{\left[1+2 s \lambda_{0} \log \left(\frac{\Lambda}{u\left(\vec{p}_{3}, \vec{p}_{4}\right)}\right)\right]^{2} f_{p_{1}} f_{p_{2}} f_{p_{3}} f_{p_{4}}}=0
\end{aligned}
$$

All of the remaining diagrams will not contribute to the renormalized kinetic equation because each of the more complex diagram will have the lines going in the opposite direction and we shall always encounter expressions like (6.12) while calculating them.

Let us begin with the integrating out the momentum which goes around the minimal loop (and the minimal loop is the one which goes just between two vertices). Then we shall obtain the diagram where this loop is replaced by a dot multiplied by the logarithm of $\Lambda$. Really, the expression for the diagram came from the sum over different trees. If the tree went alone one of the propagators of the loop, then the the loop momentum should enter the expression in the form of a multiplier (5.5) or (5.6) depending on whether this loop has parallel or opposite arrows. The multiplier (5.5) is integrated out to give $\log (\Lambda)$ while 
the (5.6) gives the finite answer. What is left after the integration is exactly the very same diagram with that loop shrunk to a point. As for the trees not going through the loop, the integration over the loop momentum gives the convergent integral as those trees do not give rise to the inverse loop propagator $f$ in the numerator (we remember that the $f$ 's in the numerator are taken from the trunk of the tree) thus making the integral convergent. If we continue this procedure shrinking the minimal loops one by one, sooner or later we encounter the loops with the opposite arrows (5.6) and the integral will not acquire the necessary power of the logarithm. The only exception to this case is the diagrams shown on fig. 3. That is why they are the only diagrams we need to sum up. Of course, there is an IR problem left. After integrating over the minimal loop, we are left with not only the logarithms, but also with the positive powers of the external momenta due to the IR terms 6.11) (or additional logarithms if the system transports waves instead of energy). They can lead to divergent integrals in subsequent integrations. For now, we just neglect those IR terms.

Now our task is to find such $f_{p}$ which solve the equation (6.14). We should look for $f_{p}$ in the form of (6.5). If we plug it into (6.14), we can discover that the values of the coefficients $c_{n}$ depend crucially on the form of the interaction (except for $c_{1}$ ). Fortunately, it is not the exact form of $f_{p}$ that we are looking for. We are interested in the asymptotic behavior of $f_{p}$ for large $\log (\Lambda / p)$. This behavior can be shown to be universal. It is more or less clear even without any computations. (6.14) has an additional $\log ^{2}(\Lambda)$ in the denominator which should be canceled by the additional log in $f$. That implies that the asymptotic behavior of $f$ is just

$$
f_{p}=p^{\gamma} \log ^{-\frac{2}{3}}\left(\frac{\Lambda}{p}\right)
$$

The proof of $(6.15)$ is rather messy and not instructive at all, so we shall not give it while noting that it exists.

We can summarize the result obtained as follows. If $\alpha<1$, the interaction is chosen in the form (45), and $\kappa=0$, then the asymptotic behavior of the inverse wave density $f_{p}$ is given by (6.15). If $\kappa$ is a small negative number, then the asymptotic behavior of $f_{p}$ will be

$$
f_{p}=p^{\gamma-\frac{2}{3} \kappa}
$$

and the energy spectrum density will be

$$
E_{p}=p^{\alpha-\gamma+\frac{2}{3} \kappa}
$$

The spectrum was modified by $\frac{2}{3} \kappa$ which is called the anomalous dimension. 


\section{Conclusions and open problems}

So we have shown how one can deal with the turbulence correlation functions for the system with a small interaction. There is a task of classifying the IR contributions to the self energy and other corrections not accomplished here, but it seems the technique we developed is powerful enough for this work to be done in the nearest future.

However, the most challenging problem to solve would be to find the correlation functions for the systems described by the Hamiltonian

$$
H_{h}=\sum_{p_{1} p_{2} p_{3} p_{4}} \lambda_{p_{1} p_{2} p_{3} p_{4}} a_{p_{1}}^{\dagger} a_{p_{2}}^{\dagger} a_{p_{3}} a_{p_{4}}
$$

With a particular choice of $\lambda$ this Hamiltonian describes the motion of the incompressible fluid (see ref. [1]). This theory possesses the integrals $F$ in the very same fashion as the theory (1.1). Unfortunately we know of no way to find them explicitly as the equations of motion which follow from (7.1) are impossible to solve. Still some of their properties can probably be studied by extrapolating the properties of (2.2) even though the most pointblank extrapolation, that is $\omega \rightarrow 0$, is most probably not possible as a phase transition may take place.

It would also be especially interesting to study these probability distributions in two dimensions. We may expect them to give rise to certain conformal field theories with unusual properties. The distribution (2.2) is essentially nonlocal even for the initially local Hamiltonian while the actions considered in conformal field theory are usually supposed to be local. And while the distribution (2.2) is explicitly real, the theory it describes can naturally be nonunitary because of the possible anomaly in the wave number conservation. The idea of the conformal invariance of the turbulence correlation functions was put forward and exploited in [8] for the case of hydrodynamic turbulence but perhaps the study of the differences of the standard statistical distributions and the turbulent distributions can shed more light on this subject. It is also perhaps possible to formulate a simple statistical model like Ising model with probability distributions similar to (2.2) and study its properties by direct computation.

The author is grateful to A.M. Polyakov for sharing his ideas and especially for his support and interest in this work and to I. Kogan for many very useful discussions. 


\section{References}

[1] V. Zakharov, V. L'vov and G. Falkovich, "Kolmogorov spectra of turbulence I", Springer-Verlag Berlin Heidelberg, (1992)

[2] A.Polyakov, unpublished

[3] H. W. Wyld, Ann. Phys. 14 (1961) 143

[4] V.E. Zakharov and V.S. L'vov, Radiophysics and Quantum Electronics 18(10) (1975) 1084

[5] V. Erofeev and V. Malkin, Zh.E.T.F. 96(5) (1989) 1666 [English translation: Sov. Phys. JETP 69(5) (1989) 943]

[6] A. Patashinski and V.Pokrovski, "Fluctuation Theory of Phase Transitions", Pergamon Press, Oxford (1979)

[7] J. Kogut and K. Wilson, Phys. Rep. 12 (1974) 75

[8] A. Polyakov, Nucl. Phys. B396 (1993) 367 